\title{
Tipos polínicos encontrados em esfingídeos (Lepidoptera, Sphingidae) em área de Floresta Atlântica do Sudeste do Brasil: uso da palinologia no estudo de interações ecológicas ${ }^{1}$
}

\author{
RUBEM SAMUEL DE AVILA JUNIOR ${ }^{2,4}$, MARIA AMÉLIA VITORINO DA CRUZ-BARROS ${ }^{3}$, \\ ÂNGELA MARIA DA SILVA CORREA ${ }^{3}$ e MARLIES SAZIMA ${ }^{2}$
}

(recebido: 19 de novembro de 2009; aceito: 04 de junho de 2010)

\begin{abstract}
Pollen types found on hawkmoths (Lepidoptera, Sphingidae) in the Atlantic Rain Forest area of Southeastern Brazil: use of palinology in studies of ecological interactions). The species of the plants used by a hawkmoth community (Lepidoptera, Sphingidae) were recorded through pollen types collected on the bodies of specimens in an area of the Atlantic Rain Forest of Southeastern Brazil. A total of 63 pollen types of 27 families were recorded, which showed assimetrical temporal distribution during the study. Species of Rubiaceae, Bromeliaceae and Fabaceae predominated in the records. The Inga pollen type was present in almost all the recorded months and in many of the hawkmoths species. Pollen types of species that did not present sphingophilous traits were the most common in the samples, a fact that could be related with the generalist foraging pattern of this group of Lepidoptera. This study demonstrates the utility of palinology on ecological studies of plant-pollinator interactions in the tropics.
\end{abstract}

Key words - generalist foraging, hawkmoth community, Inga, Rubiaceae

RESUMO - (Tipos polínicos encontrados em esfingídeos (Lepidoptera, Sphingidae) em área de Floresta Atlântica do Sudeste do Brasil: uso da palinologia no estudo de interações ecológicas). Foram determinadas as espécies de plantas utilizadas por uma comunidade de esfingídeos (Lepidoptera, Sphingidae) através da avaliação dos tipos polínicos que os espécimes apresentavam sobre seus corpos, em uma área de Floresta Atlântica do Sudeste do Brasil. Foram registrados 63 tipos polínicos de 27 famílias distribuídos assimetricamente ao longo do ano. Houve predomínio de espécies de Rubiaceae, Bromeliaceae e Fabaceae. Pólen do tipo Inga esteve presente em quase todos os meses amostrados e em grande número de espécies de esfingídeos. Tipos polínicos relativos a famílias que não apresentam atributos morfológicos adaptativos ao uso por Sphingidae foram responsáveis pela maior parte das amostras, o que demonstra forte aspecto generalista no comportamento de forrageamento deste grupo de lepidópteros. Este estudo reforça a utilidade do uso da palinologia no entendimento de padrões de interações inseto-planta em ambientes tropicais.

Palavras-chave - comportamento generalista, comunidade de mariposas, Inga, Rubiaceae

\section{Introdução}

Mariposas da família Sphingidae (Lepidoptera) constituem um grupo taxonômico bastante diverso ( $c a$. de 1.200 spp.) com ampla distribuição mundial (Kitching \& Cadiou 2000) possuindo características utilizáveis no biomonitoramento da qualidade ambiental de ecossistemas (Hilty \& Merenlender 2000). Soma-se a isso, o fato de serem agentes polinizadores de numerosas espécies em ecossistemas tropicais (Bawa 1990, Oliveira 1991),

1. Parte da tese de doutorado do primeiro autor, Programa de Pós-Graduação em Biologia Vegetal, Universidade Estadual de Campinas, Campinas, SP, Brasil.

2. Universidade Estadual de Campinas, Departamento de Biologia Vegetal, Instituto de Biologia, Caixa Postal 6109, 13083-970 Campinas, SP, Brasil.

3. Instituto de Botânica de São Paulo, Núcleo de Pesquisa em Palinologia, Caixa Postal 3005, 01031-970 São Paulo, SP, Brasil.

4. Autor para correspondência: rubemavila@yahoo.com.br tendo importante papel na estruturação e na dinâmica de populações vegetais. Esta função poderia ser avaliada e medida, por exemplo, pelo número de espécies vegetais que dependem do serviço de polinização deste grupo de insetos. Porém, esfingídeos apresentam comportamento quase exclusivamente noturno, o que torna a observação das visitas às flores bastante restrita e, consequentemente, a avaliação de sua importância no sistema é dificultada, baseando-se quase que totalmente em taxas de sucesso reprodutivo nas espécies que apresentam atributos de polinização por esfingídeos: flores de coloração branca, antese noturna e que emitem odor adocicado são alguns exemplos. Este fato não ocorre em sistemas de interações diurnas (plantas e beija-flores ou plantas e abelhas) que podem ser facilmente observadas e avaliadas quanto à eficiência do polinizador. Neste sentido, a utilização de outras metodologias em estudos reprodutivos de espécies vegetais esfingófilas, principalmente em nível de comunidade, torna-se fundamental (Palmer et al. 2003, Alarcón et al. 2008). 
A evidência de visitas às flores por parte do polinizador/visitante pode ser indiretamente obtida pela presença de pólen em partes de seu corpo (Kislev et al.1972, Singer \& Cocucci 1997, Alexandersson \& Johnson 2002, Nattero et al. 2003, Marques-Souza et al. 2007). Neste sentido, a associação da ecologia da polinização e da palinologia é de extrema importância, porém, poucos estudos com esfingídeos têm utilizado essa associação em nível comunitário (Haber \& Frankie 1989, Darrault \& Schlindwein 2002, Alarcón et al. 2008). Embora nem sempre a associação possibilite interpretar a eficiência do polinizador, fornece informações para a confirmação de possíveis interações não registradas devido às dificuldades da observação noturna.

Este estudo teve como objetivos responder as seguintes perguntas: i) Qual é a composição de espécies de plantas utilizadas como recurso floral da comunidade de esfingídeos (Lepidoptera, Sphingidae) em áreas da Floresta Atlântica do Parque Estadual da Serra do Mar? ii) As espécies encontradas refletem as características de esfingofilia? iii) Existem espécies de plantas mais importantes e como estão distribuídas temporalmente? Além disso, esse estudo teve como objetivo apontar a utilização da amostragem de tipos polínicos como indicadores da interação planta-polinizador.

\section{Material e métodos}

O estudo foi realizado em área de Floresta Atlântica no Sudeste do Brasil (Parque Estadual da Serra do Mar, Núcleo Picinguaba, Ubatuba, $23^{\circ} 20^{\prime}-23^{\circ} 22^{\prime} \mathrm{S}$; $44^{\circ} 46^{\prime}-44^{\circ} 51^{\prime} \mathrm{W}$ ) ao longo de um ano de coletas mensais (agosto de 2005 a julho de 2006). Os esfingídeos foram coletados por meio de armadilha luminosa em noites de lua nova e identificados com bibliografia especializada (D'Abrera 1986, Kitching \& Cadiou 2000, Moré et al. 2005). O pólen encontrado em diferentes partes do corpo dos espécimes capturados foi coletados e armazenado em ácido acético glacial. A preparação do material polínico foi feita segundo o método de acetólise de Erdtman (1960). Os grãos de pólen foram submetidos à ação de solução de anidrido acético e ácido sulfúrico em banho-maria à temperatura de aproximadamente $100{ }^{\circ} \mathrm{C}$, a seguir foram montados em lâminas com gelatina glicerinada. Posteriormente foi feita a análise taxonômica dos tipos polínicos com base na coleção de referência da Palinoteca do Núcleo de Pesquisas em Palinologia do Instituto de Botânica e em literatura especializada (Melhem et al. 1984, Roubik \& Moreno 1991). As lâminas com o material polínico estão depositadas na referida palinoteca do Instituto de Botânica de São Paulo. Os tipos polínicos foram contabilizados quanto à sua freqüência em cada mês e foi estabelecido um valor de importância para cada espécie/tipo polínico relativo à sua proporcionalidade no total da flora $\mathrm{e}$ quanto a sua incidência na comunidade de esfingídeos. Esta metodologia foi estabelecida com o intuito de minimizar os possíveis efeitos das diferentes cargas polínicas de cada espécie e as possíveis presenças acidentais na amostra. Assim, adotaram-se os seguintes parâmetros:

$$
\begin{aligned}
& \text { Índice de proporcionalidade (ip) }=\frac{\mathrm{n}^{\mathrm{o}} \text { de grãos-de-pólen da espécie } i}{\mathrm{n}^{\mathrm{o}} \text { total de grãos-de-pólen das }} \times 100 \\
& \text { spp do mês } j
\end{aligned}
$$

$\begin{aligned} & \text { Índice de } \\ & \text { ocorrência (io) }\end{aligned}=\frac{\text { no de spp. de esfingídeos com presença de pólen } i}{\mathrm{n}^{\circ} \text { total de estingídeos do mês } j}$

Valor de importância $(v i)=$ ip $\times$ io

\section{Resultados}

Nas 49 espécies de Sphingidae amostradas houve registro de 64 tipos polínicos de 27 famílias de angiospermas, com predomínio de espécies de Rubiaceae $(15,6 \%)$, Bromeliaceae $(7,8 \%)$ e Fabaceae $(6,2 \%)$ ) figuras 1 a 39). Maior número de espécies/tipos polínicos foi registrado nos meses de novembro (20 spp.), outubro (19 spp.), dezembro (16 spp.), janeiro (15 spp.) e fevereiro (13 spp.) (tabela 1). Das 27 famílias registradas, apenas sete apresentam informações de interações com esfingídeos (Apocynaceae, Asteraceae, Convolvulaceae, Malvaceae, Fabaceae, Scrophulariaceae e Rubiaceae). Os tipos polínicos de Bromeliaceae, Arecaceae, Begoniaceae, Celastraceae, Combretaceae, Cyperaceae, Erythroxylaceae, Malvaceae, Moraceae, Melastomataceae, Piperaceae, Poaceae, Polygonaceae, Sapotaceae, Scrophulariaceae, Solanaceae, Ulmaceae e Verbenaceae (figuras 1 a 39) tiveram seu primeiro registro como fonte de recursos florais de esfingídeos no presente estudo. Apesar da alta diversidade de táxons registrados, a distribuição temporal apresentou grande variação no número de espécies havendo meses com cinco tipos polínicos e outros com 20 tipos polínicos encontrados nos esfingídeos (tabela 1).

Quanto à importância dos táxons vegetais nas interações com esfingídeos, foi observada a relação entre o índice de proporcionalidade (ip) e o valor de importância (vi) para todos os meses amostrados (Correlação de Pearson, $\mathrm{r}=0,55, P=0,001)$ nos quais as espécies com maior ip foram também as espécies com maior valor de vi (tabela 1). Porém, foi observada a existência de casos em que o tipo polínico mais abundante, ou seja, com maior valor de ip, não foi o mais freqüente nas espécies de esfingídeos em determinado mês, diminuindo assim, o seu vi. Este fato ocorreu nos meses de janeiro, fevereiro, março e outubro (tabela 1).

A estimativa do valor de importância (vi) reflete a associação entre abundância do tipo polínico e sua 

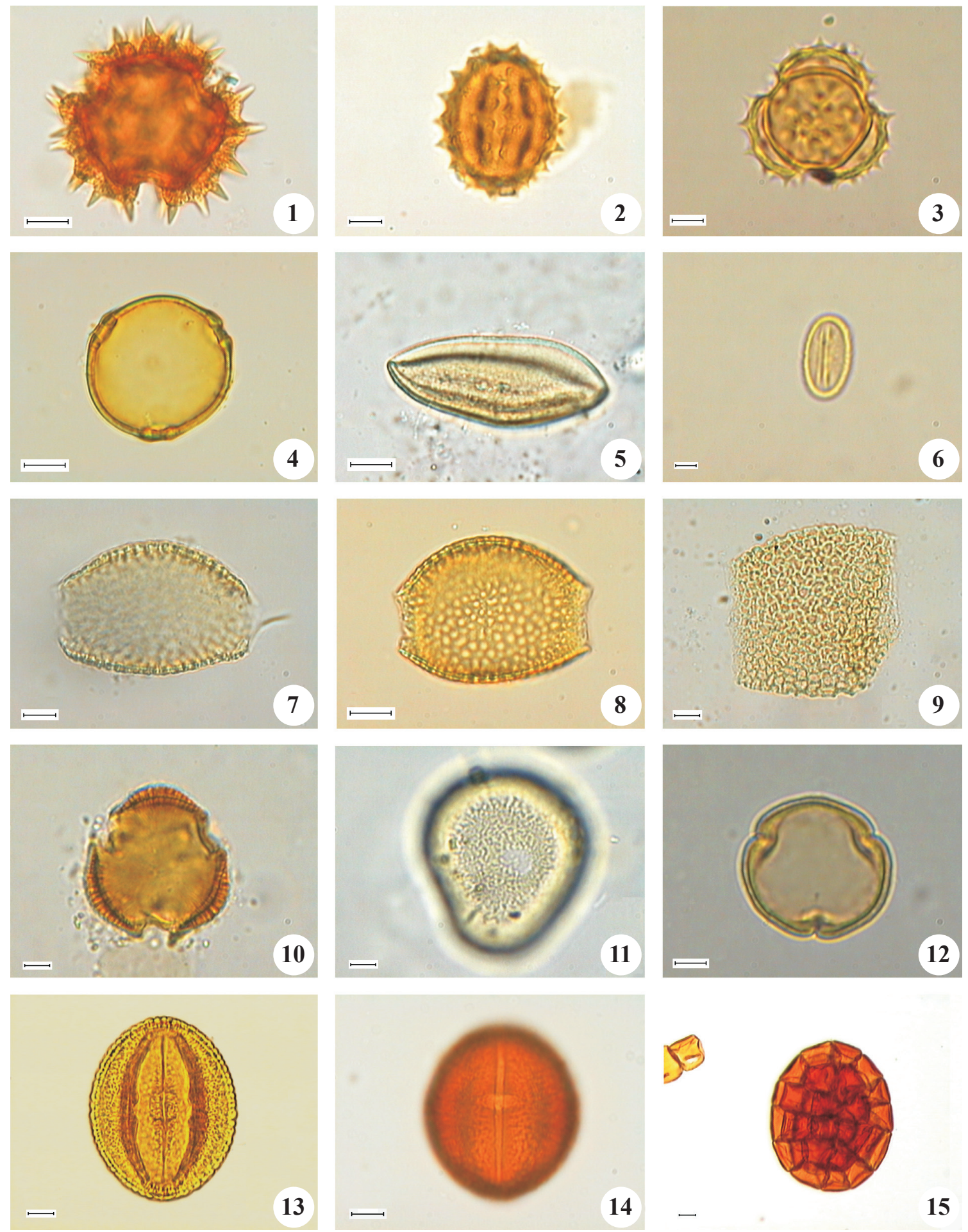

Figuras 1-15. Tipos polínicos encontrados em esfingídeos. 1-3. Asteraceae. 1. Vernonia sp.2. Asteraceae sp.1. 3. Asteraceae sp.2. 4. Apocynaceae. Peltastes peltatus. 5. Arecaceae. 6. Begoniaceae. Begonia sp. 7-9. Bromeliaceae. 7. Aechmea sp. 8. Neoregelia sp. 9. Nidularium sp. 10. Celastraceae. Maytenus sp. 11. Cyperaceae. 12-13. Euphorbiaceae. 12. Pera sp. 13. Sapium sp. 14. Erythroxylaceae. Erythroxylum sp. 15. Fabaceae. Inga sp. Barra $=10 \mu \mathrm{m}(1-2,4-5,7-8,13-14) ; 5 \mu \mathrm{m}(3,6,9-12) ; 20 \mu \mathrm{m}(15)$.

Figures 1-15. Pollen types founded on hawkmoths. 1-3. Asteraceae. 1. Vernonia sp.2. Asteraceae sp.1. 3. Asteraceae sp.2. 4. Apocynaceae. Peltastes peltatus. 5. Arecaceae. 6. Begoniaceae. Begonia sp. 7-9. Bromeliaceae. 7. Aechmea sp. 8. Neoregelia sp. 9. Nidularium sp. 10. Celastraceae. Maytenus sp. 11. Cyperaceae. 12-13. Euphorbiaceae. 12. Pera sp. 13. Sapium sp. 14. Erythroxylaceae. Erythroxylum sp. 15. Fabaceae. Inga sp. Bar $=10 \mu \mathrm{m}:(1-2,4-5,7-8,13-14) ; 5 \mu \mathrm{m}(3,6,9-12) ; 20 \mu \mathrm{m}(15)$. 


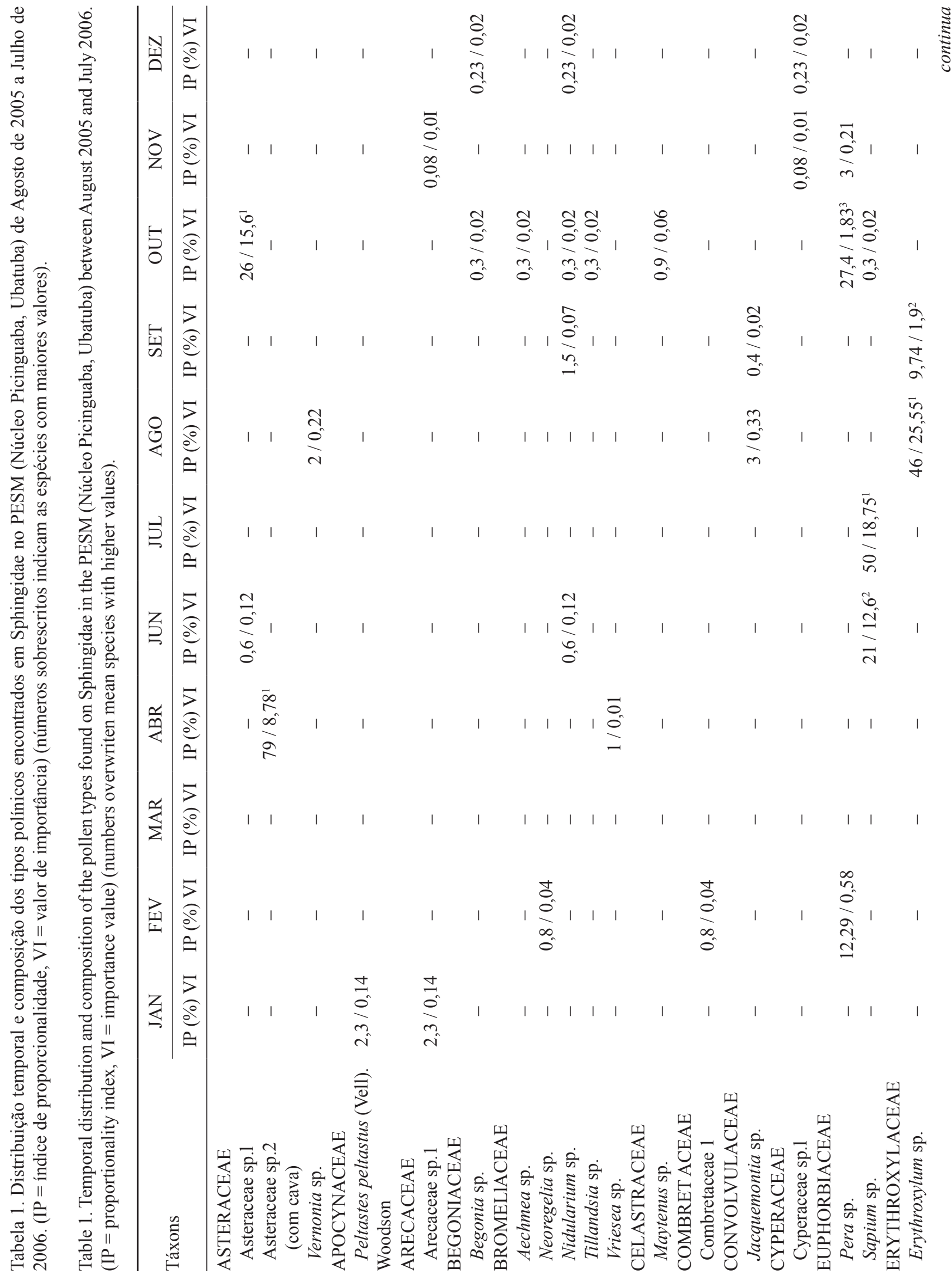









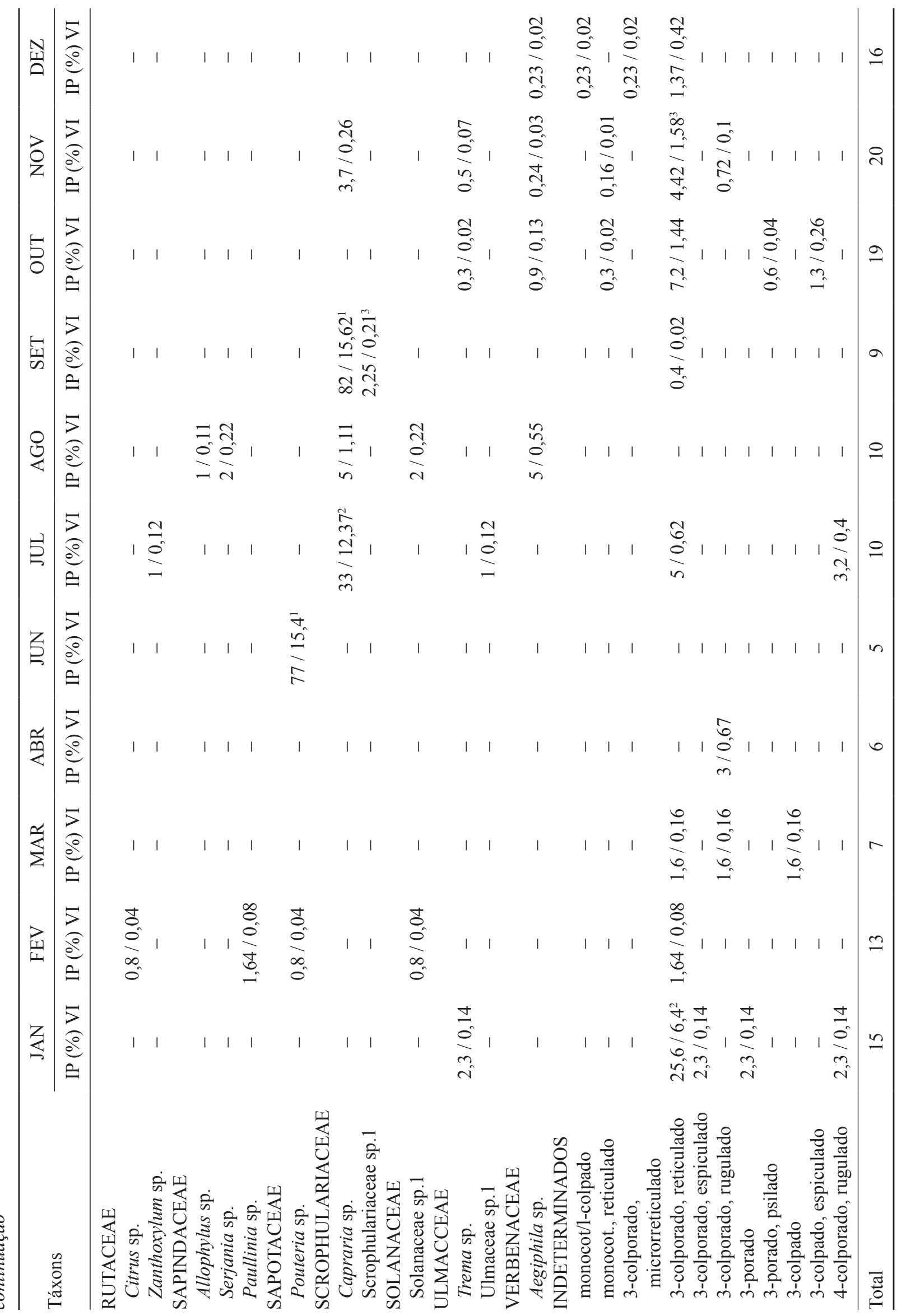


distribuição na comunidade de esfingídeos. O tipo polínico Inga (figura 15) apresentou maiores valores deste atributo em três dos 11 meses de ocorrência de esfingídeos (janeiro, fevereiro e março) e, ainda, o segundo maior valor deste parâmetro em outros dois meses. Guettarda viburnoides Cham. \& Schltdl. (figura 30) apresentou o maior valor de vi em dois meses (novembro e dezembro) (tabela 1). Os tipos polínicos das
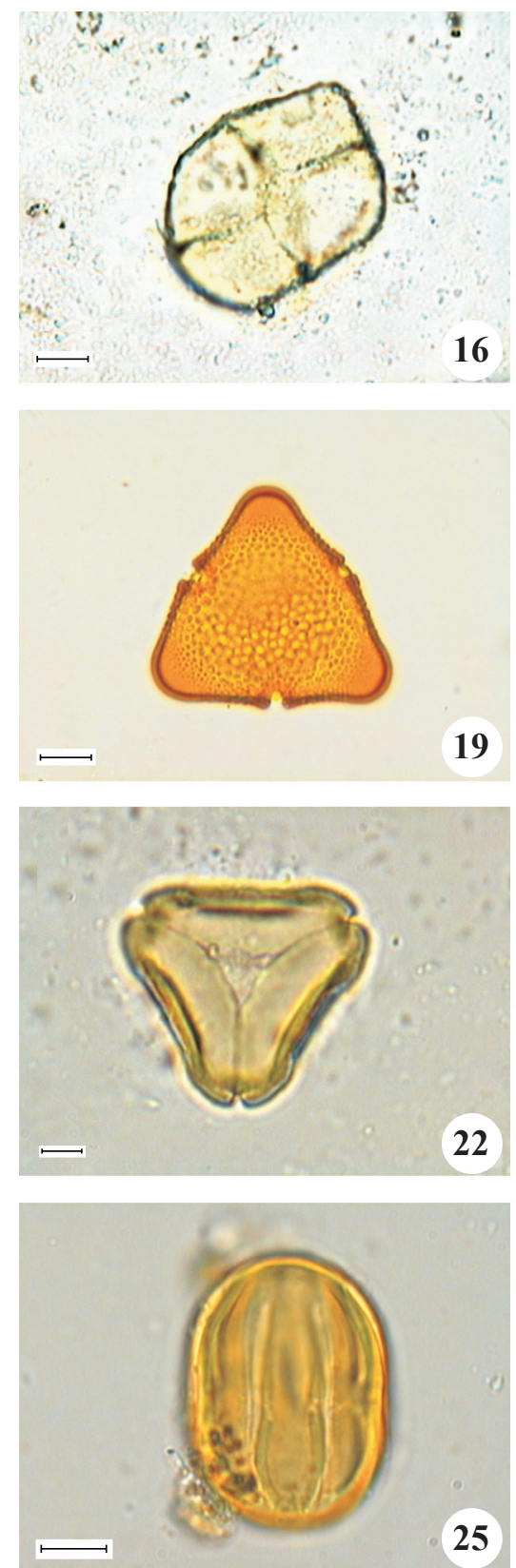

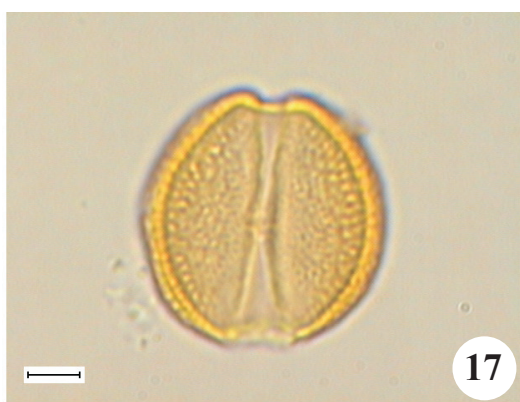

17

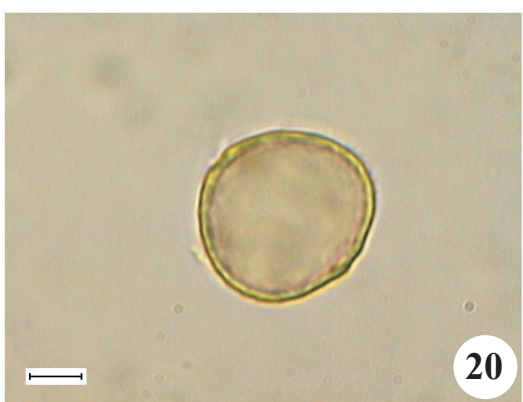

20
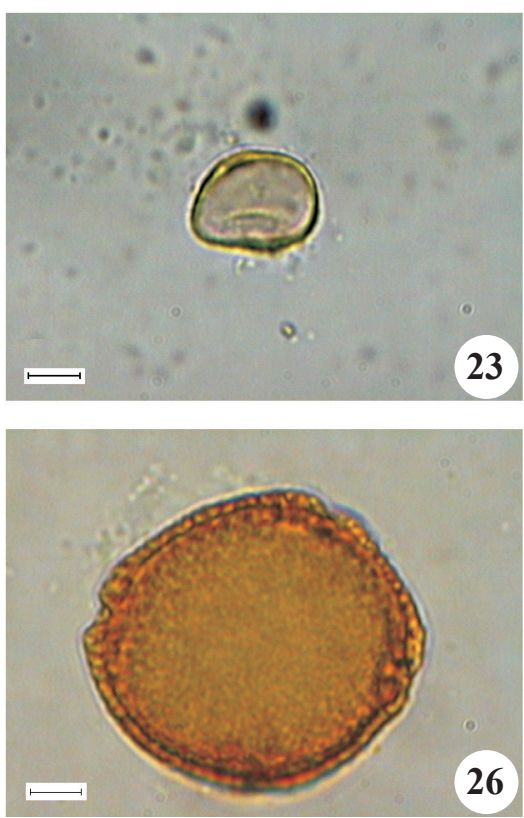
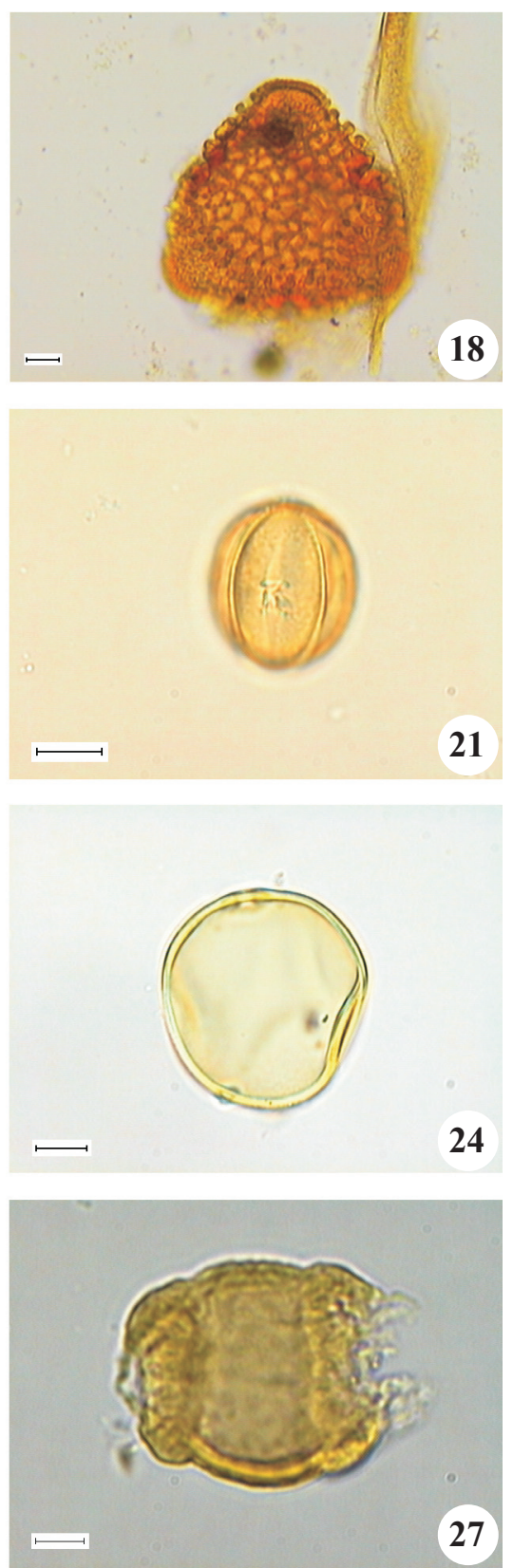

Figuras 16-27. Tipos polínicos encontrados em esfingídeos. 16-17. Fabaceae. 16. Mimosa selloii. 17. Stylosanthes sp. 18-19. Malvaceae. 18. Bombacopsis sp. 19. Pseudobombax sp. 20. Moraceae. Sorocea sp. 21. Melastomataceae. 22. Myrtaceae. 23. Piperaceae. 24. Poaceae. 25. Polygalaceae. Diclidanthera sp. 26-27. Rubiaceae. 26. Alibertia sp. 27. Coussarea sp. Barra = $10 \mu \mathrm{m}(16,18-19,21,24-25) ; 5 \mu \mathrm{m}(17,20,22-23,26-27)$.

Figures 16-27. Pollen types founded on hawkmoths. 16-17. Fabaceae. 16. Mimosa selloii. 17. Stylosanthes sp. 18-19. Malvaceae. 18. Bombacopsis sp. 19. Pseudobombax sp. 20. Moraceae. Sorocea sp. 21. Melastomataceae. 22. Myrtaceae. 23. Piperaceae. 24. Poaceae. 25. Polygalaceae. Diclidanthera sp. 26-27. Rubiaceae. 26. Alibertia sp. 27. Coussarea sp. Bar $=10 \mu \mathrm{m}(16,18-19$, 21, 24-25); 5 um (17, 20, 22-23, 26-27). 
18 famílias que tiveram seu primeiro registro como fonte de recursos florais de esfingídeos neste estudo, estão representados com os menores valores de ip e vi.

Dos tipos polínicos amostrados, 54\% tiverem ocorrência restrita a um mês, $22,2 \%$ foram observados em dois meses, $9,5 \%$ em três meses e $14,5 \%$ foram registrados nos espécimes de esfingídeos em quatro ou mais meses da amostragem. O gênero Inga (figura 15) foi o tipo polínico com maior freqüência nas amostras, ocorrendo em nove dos onze meses de incidência de
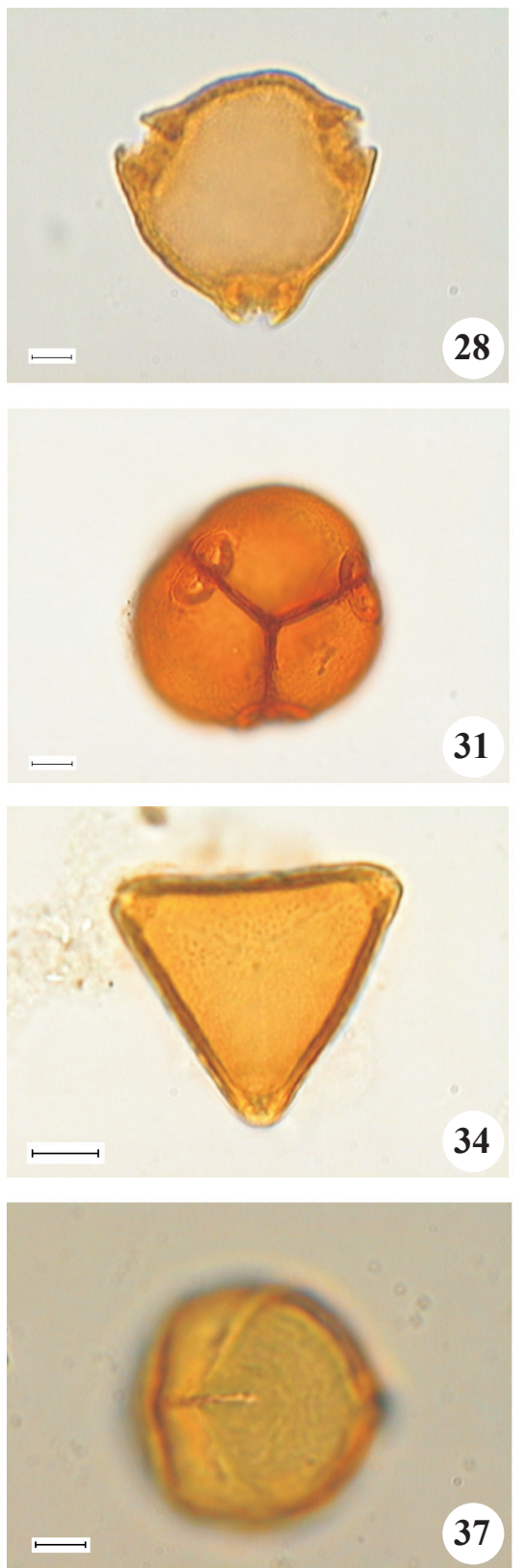

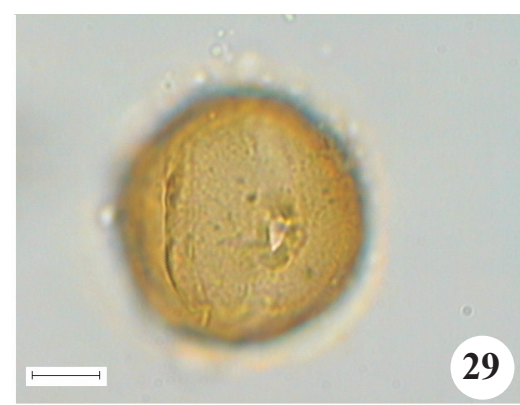

29
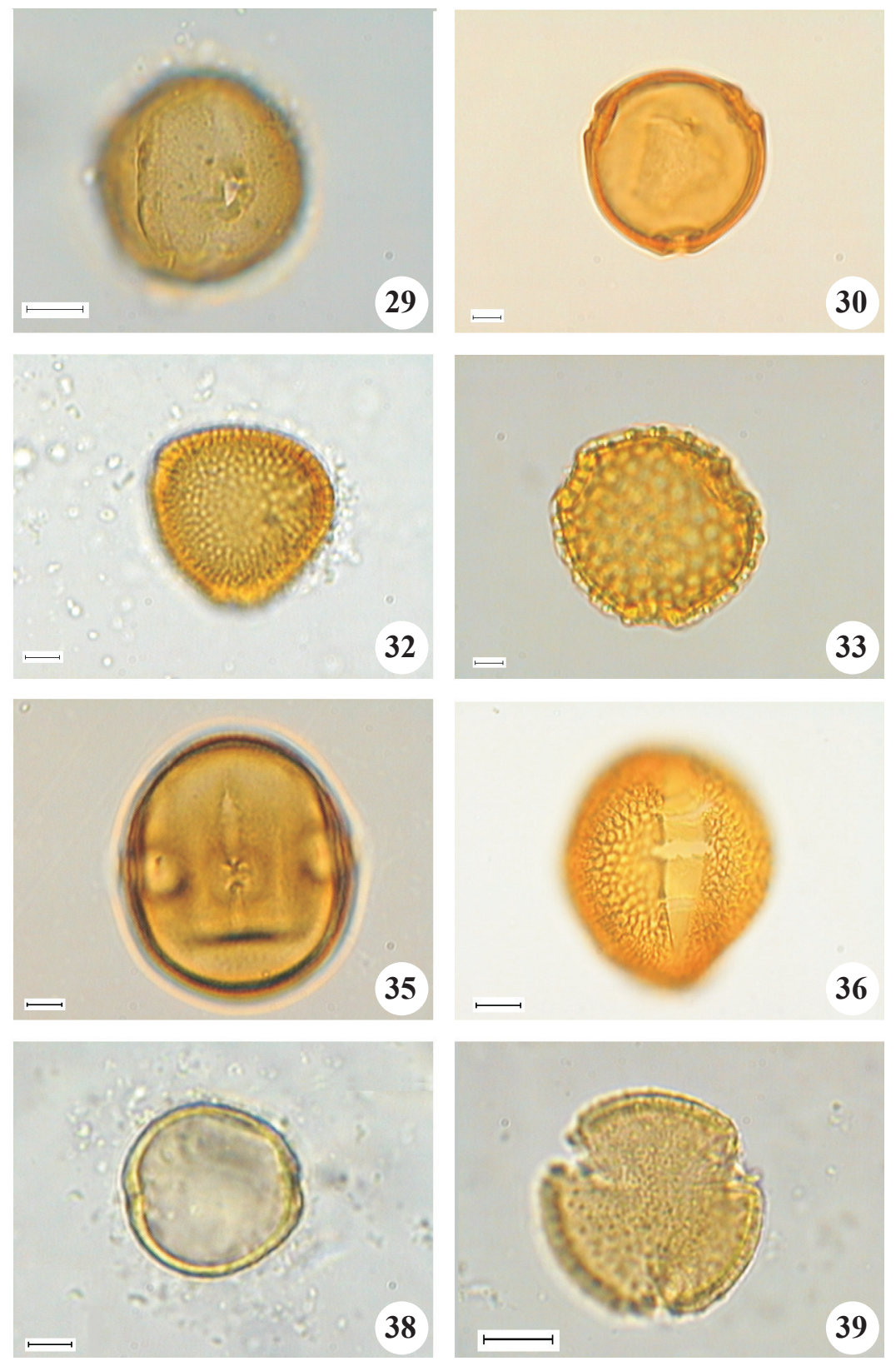

Figuras 28-39. Tipos polínicos encontrados em esfingídeos. 28-33. Rubiaceae. 28. Faramea sp. 29. Genipa infundibuliformis. 30. Guettarda viburnoides. 31. Randia sp. 32. Sabicea sp. 33. Tocoyena sp. 34. Sapindaceae. Serjania sp. 35. Sapotaceae. Pouteria sp. 36. Scrophulariaceae. Capraria sp. 37. Solanaceae. 38. Ulmaceae. Trema sp. 39. Verbenaceae. Aegiphila sp. Barra = $10 \mu \mathrm{m}(29,31,34,36,39) ; 5 \mu \mathrm{m}(28,30,32-33,35,37-38)$.

Figures 28-39. Pollen types founded on hawkmoths. 28-33. Rubiaceae. 28. Faramea sp. 29. Genipa infundibuliformis. 30. Guettarda viburnoides. 31. Randia sp. 32. Sabicea sp. 33. Tocoyena sp. 34. Sapindaceae. Serjania sp. 35. Sapotaceae. Pouteria sp. 36. Scrophulariaceae. Capraria sp. 37. Solanaceae. 38. Ulmaceae. Trema sp. 39. Verbenaceae. Aegiphila sp. Bar $=10 \mu \mathrm{m}$ $(29,31,34,36,39) ; 5 \mu \mathrm{m}(28,30,32-33,35,37-38)$. 
esfingídeos. Ainda com ampla distribuição temporal foi registrado o tipo polínico 3-colporado, reticulado (oito meses), pólen das famílias Myrtaceae e Poaceae (seis meses), Melastomataceae (cinco meses) e da Rubiaceae Guettarda viburnoides (quatro meses) (tabela 1 e figuras $21,22,24$ e 30 ). No mês de maio não houve incidência de esfingídeos na amostra.

\section{Discussão}

O predomínio de tipos polínicos da família Rubiaceae neste estudo confirma os dados de Darrault \& Schlindwein (2002) em região de cerrado do nordeste do Brasil. Embora com fitofisionomias bastante distintas, a prevalência de espécies desta família nos dois estudos aponta para a grande convergência de características florais adaptadas à utilização de esfingídeos como visitantes/polinizadores em Rubiaceae. Alguns estudos sobre biologia da polinização baseados em características florais têm apontado uma convergência de caracteres florais compatíveis com a polinização por esfingídeos nesta família tais como, corola hipocrateriforme ou infundibuliforme com coloração branca ou amarelada da mesma (Haber \& Frankie 1989, Vogel 1990). Espécies de Fabaceae (Mimosoidae) também são conhecidas pela associação com esfingídeos (Haber \& Frankie 1989). Pólen de duas espécies deste grupo taxonômico, Mimosa dysocarpa Benth. e Calliandra eryophylla Benth. também foram encontradas com alta freqüência em esfingídeos em uma área do hemisfério norte (Alarcón et al. 2008), corroborando a importância deste grupo taxonômico como fonte de recurso alimentar para esfingídeos. Por outro lado, a alta representatividade de espécies de Rubiaceae e Fabaceae na amostragem polínica neste estudo poderia refletir o fato de ambas serem famílias com alta diversidade em Floresta Atlântica (Sanchez et al. 1999) além de habitualmente abundantes (Alarcón et al. 2008). Além disso, representantes das famílias Apocynaceae, Asteraceae, Convolvulaceae, Euphorbiaceae, Myrtaceae e Sapindaceae também foram mencionados por Darrault \& Schlindwein (2002) em região de cerrado do nordeste brasileiro.

Os menores valores de ip e vi dos tipos polínicos das 18 famílias que tiveram seu primeiro registro como fonte de recursos florais de esfingídeos neste estudo, associados ao fato de que alguns destes grupos taxonômicos são caracterizados por flores com quantidades mínimas de recursos, podem ser um forte indício de que as interações tenham ocorrido acidentalmente, ocasionando interações indiretas (Alarcón et al. 2008). Algumas espécies registradas no estudo oferecem apenas pólen como recurso, como ocorre com espécies de Begoniaceae, por exemplo. Embora as interações indiretas possam ocorrer, vale ressaltar que este grupo de macrolepidoptera possa estar utilizando pólen em sua dieta à semelhança de algumas espécies de lepidópteros diurnos que apresentam adaptações morfofisiológicas que auxiliam no incremento de nitrogênio em sua dieta nectarífera com pólen (Gilbert 1972, Brown 1981, Eberhard et al. 2009).

Os maiores valores dos atributos medidos (ip e vi) encontrados para Inga e Guettarda neste estudo condizem com os dados de Darrault \& Schlindwein (2002), confirmando o papel chave das espécies destes grupos nestas comunidades de esfingídeos. Porém, a função de espécies de Inga na associação com a esfingofauna de Picinguaba pode estar superestimada devido às dificuldades de identificação do pólen em nível específico, pois na área ocorrem no mínimo três espécies (obs. pess.) e que estão sendo consideradas como um único tipo polínico.

A grande riqueza de táxons de plantas registrados evidencia o valor de estudos palinológicos relacionados às interações planta-polinizador e confirma que $o$ comportamento de forrageamento de várias espécies de esfingídeos apresenta padrão generalista quanto ao uso de recursos florais, aspecto oposto às interações mais específicas que o conceito de síndrome de polinização prediz (Faegri \& van der Pijl 1971). Padrão generalista nas interações mutualísticas tem sido mencionado em diferentes sistemas planta-polinizador (Jordano 1987, Waser et al. 1996, Gómez \& Zamora 1999) e particularmente para uma espécie de esfingídeo (Kislev et al.1972). No entanto, vale ressaltar que os dados obtidos neste estudo fazem inferência à amplitude de utilização de recurso alimentar por parte da comunidade de esfingídeos, não indicando, necessariamente, que estes sejam utilizados pelas espécies vegetais como polinizadores.

Agradecimentos - Ao Núcleo de Desenvolvimento Picinguaba e ao Instituto Florestal, pela concessão de licença de pesquisa; a G. S. Girardi e M. Pinheiro, pelo auxílio nos trabalhos de campo; à Fapesp (bolsa para R. S. Avila Jr., proc. nº 04/14354-0 e Auxílio à Pesquisa para M. Sazima, proc. no 05/01354-4) e ao CNPq. Este trabalho também foi financiado pela Fapesp no âmbito do Projeto Temático Gradiente Funcional (Processo 03/12595-7), que faz parte do Programa Biota/Fapesp - O Instituto Virtual da Biodiversidade. Autorização Cotec/IF 41.065/2005 e autorização Ibama/CGEN 093/2005. 


\section{Referências bibliográficas}

ALARCÓN, R., DAVIDOWITZ, G. \& BRONSTEIN, J. 2008. Nectar usage in a southern Arizona hawkmoth community. Ecological Entomology 33:503-509.

ALEXANDERSSON, R. \& JOHNSON, S.D. 2002. Pollinator-mediated selection on flower-tube length in a hawkmoth-pollinated Gladiolus (Iridaceae). Proceedings of the Royal Society of London B 269:631-636.

BAWA, K.S. 1990. Plant-pollinator interactions in the Tropical Rain Forest. Annual Review of Ecology and Systematics 21:399-422.

BROWN, K.S. 1981. The biology of Heliconius and related genera. Annual Review of Entomology 26:427-456.

D'ABRERA, B. 1986. Sphingidae mundi - Hawkmoths of the world. Classey Books, Faringdon.

DAFNI, A. 1992. Pollination ecology - a practical approach. Oxford University Press, Oxford.

DARRAULT, R. \& SCHLINDWEIN, C. 2001. Esfingídeos (Lepidoptera, Sphingidae) no tabuleiro paraibano, nordeste do Brasil: abundância, riqueza e relação com plantas esfingófilas. Revista Brasileira de Zoologia 19:429-443.

EBERHARD, S.H., NEMESCHKAL, H.L. \& KRENN, H.W. 2009. Biometrical evidence for adaptations of the salivary glands to pollen feeding in Heliconius butterflies (Lepidoptera: Nymphalidae). Biological Journal of the Linnean Society 97:604-612.

ERDTMAN, G. 1960. The acetolisys method in a revised description. Svensk Botanisk Tidskrift 54:561-564.

FAEGRI, K. \& VAN DER PIJL, L. 1971. The principles of pollination ecology. Pergamon Press, New York.

GILBERT, L.E. 1972. Pollen feeding and reproductive biology of Heliconius butterflies. Proceedings of the National Academy of Sciences of the United States of America 69:1403-1407.

GÓMEZ, J.M. \& ZAMORA, R. 1999. Generalization vs. specialization in the pollination system of Hormathophylla spinosa (Cruciferae). Ecology 80:796-805.

HABER, W.A. \& FRANKIE, G. 1989. A tropical hawkmoth community: Costa Rican dry forest Sphingidae. Biotropica 21:155-172.

HILTY, J. \& MERENLENDER, A. 2000. Faunal indicator taxa selection for monitoring ecosystem health. Biological Conservation 92:185-197.

JORDANO, P. 1987. Patterns of mutualistic interactions in pollination and seed dispersal: connectance, dependence, asymmetries, and coevolution. The American Naturalist 129:656-676.
KISLEV, M.E., KRAVITZ, Z. \& LORCH, J. 1972. A study of hawkmoth pollination by a palinological analysis of the proboscis. Israel Journal of Botany 21:57-75.

KITCHING, I. \& CADIOU, J.M. 2000. Hawkmoths of the world: an annotaded and illustraded revisionary checklist (Lepidoptera: Sphingidae). Cornell University Press, London.

MARQUES-SOUZA, A.C., ABSY, M.L. \& KERR, W.S. 2007. Pollen harvest features of the Central Amazonian bee Scaptotrigona fulcicutis Moure 1964 (Apidae: Meliponinae), in Brazil. Acta Botanica Brasilica 21: 11-20.

MELHEM, T.S., MAKINO, H., SILVESTRE, M.S.F., CRUZ, M.A.V. \& JUNG MENDAÇOLLI, S.L. 1984. Planejamento para a elaboração da "Flora Polínica da Reserva do Parque Estadual das Fontes do Ipiranga (São Paulo, Brasil)". Hoehnea 11:1-7.

MORÉ, M., SERSIC, A. \& COCCUCI, A.A. 2005. Esfingidos del Argentina. Lola, Córdoba.

NATTERO, J., MORÉ, M., SÉRSIC, A.N. \& COCUCCI, A.A. 2003. Possible tobacco progenitors share longtongued hawkmoths as pollen vectors. Plant Systematics and Evolution 241:47-54.

OLIVEIRA, P.E.A.M. 1991. Reproductive biology of woody plants in cerrado community of Central Brazil. PhD Thesis, University of St. Andrews, St. Andrews.

PALMER, T.M., STANTON, M.L. \& YOUNG, T.P. 2003. Competition and coexistence: exploring mechanisms that restrict and maintain diversity within mutualist guilds. The American Naturalist 162(supl.): 63-79.

ROUBIK, D.W. \& MORENO PATIÑO, J.E. 1991. Pollen and spores of Barro Colorado Island. Missouri Botanical Gardens Monographs in Systematic Botany, St. Louis, Missouri, v.36.

SANCHEZ, M., PEDRONI, F. \& LEITÃO-FILHO, H.F. 1999. Composição florística de um trecho de floresta ripária na Mata Atlântica em Picinguaba, Ubatuba, SP. Revista Brasileira de Botânica 22:31-42.

SINGER, R. \& COCUCCI, A.A. 1997. Eye attached hemipollinaria in the hawkmoth and settling moth pollination of Habenaria (Orchidaceae): a study o functional morphology in 5 species from subtropical South America. Botanica Acta 110:328-337.

VOGEL, S. 1990. Radiacion adaptativa del sindrome floral en las familias neotropicales. Boletin de la Academia Nacional de Ciências 59:5-30.

WASER, N.M., CHITKA, L., PRICE, M.V. \& WILLIAMS, N.M. 1996. Generalization in pollination systems, and why it matters. Ecology 77:1043-1060. 